\title{
Magnetresonanztomographie bei der kongenitalen Hüftgelenksdysplasie und -luxation
}

\author{
NMR in the management of congenital dislocation \\ of the hip
}

In der Zeitschrift für Orthopädie wird erstmals von zwei Arbeitsgruppen über die Magnet-Resonanz-Tomographie in der Diagnostik und der Therapiekontrolle bei Kindern mit kongenitaler Hüftgelenksluxation berichtet (Franke et al. 1998, Wirth et al. 1998). Erste Mitteilungen über die Möglichkeiten dieses bildgebenden Verfahrens stammen aus den Niederlanden, wurden in der angloamerikanischen Literatur veröffentlicht und liegen bereits 10 Jahre বurück (Bos et al. 1988). Hat die deutschsprachige Orthopädie - wie ihr so häufig vorgeworfen wird - diese innovative Technik übersehen und erst 10 Jahre zu spät entdeckt?

Die Antwort ist nein! Inwieweit moderne bildgebende Verfahren bei Diagnose und Therapie der Hüftdysplasie und -luxation eingesetzt werden, ist nämlich ganz wesentlich von den Gesundheitssystemen abhängig. Vor 10 Jahren war die sonographische Untersuchung der Säuglingshüfte im deutschsprachigen Raum bereits fest etabliert, während im anglo-amerikanischen Raum und auch in den Niederlanden das Verfahren bis heute umstritten diskutiert wird. Auch die kernspintomographische Untersuchung gehört bei weitem nicht zum Standard des amerikanischen Orthopäden bei Diagnose und Therapie der kongenitalen Hüftdysplasie, eher schon des amerikanischen Radiologen. Die Differenzen zwischen veröffentlichter Meinung und praktizierter Orthopädie ergeben sich daraus, daß im anglo-amerikanischen Raum die bildgebende Diagnostik überwiegend in radiologischen Kliniken oder Abteilungen durchgeführt wird. Insofern ist der so wichtige Abgleich zwischen klinischer Untersuchung und bildgebender Diagnostik häufig nicht gewährleistet. Nur so ist es zu verstehen, daß Catterall noch vor kurzem das „Rauschen der schwarzen und weißen Punkte " bei der Sonographie bemäkelt (1994) und die Bedeutung der klinischen Untersuchung hervorhebt. Innovative bildgebende Techniken wurden im anglo-amerikanischen Sprachraum nur dort weiterentwickelt, wo sich eine hervorragende Zusammenarbeit zwischen orthopädischen und radiologischen Kliniken entwickelt hat (z. B. Harke). Eine derartige Zusammenarbeit scheint auch in den beiden Kliniken vorzuliegen, die nun aus Deutschland über die Magnet-Resonanz-Tomographie bei der Repositionsbehandlung der kongenitalen Hüftgelenksluxation berichten.

\footnotetext{
Z. Orthop. 136 (1998) 195-196

1. 1998 F. Enke Verlag Stuttgart
}

Für die Magnet-Resonanz-Tomographie gelten aber gleiche Bedenken, wie sie im anglo-amerikanischen Raum dem sonographischen Screening gegenüber vorgebracht werden. Dieses hat sich dort nicht durchgesetzt, weil Orthopäden und Radiologen das Krankheitsbild unterschiedlich beurteilen. Für den einen steht der klinische Befund der Instabilität und Dislokation, für den anderen der morphologische der Reifungsverzögerung im Vordergrund. Jeder, der eine Hüftgelenksluxation behandelt hat. also im wahrsten Sinne des Wortes in der Hand gehabt hat. weiß wo die Tücken der bildgebenden Verfahren liegen. Jede Unsicherheit bei der geschlossenen Reposition oder Grenzen der Stabilität nach offener Reposition bleiben dem Operateur unverborgen. Er wird sein Behandlungsergebnis und den Behandlungserfolg immer aus der kritischen Sicht des Behandlers werten und sich nicht allein auf die bildgebende Diagnostik verlassen und sei sie noch so gut.

Daran vermag auch die Magnet-Resonanz-Tomographie zunächst keine wesentliche Änderung herbeizuführen. Wirth et al. betonen, daß es notwendig sei, die Zahl der Reluxationen möglichst klein zu halten und die eingetretene Reluxation sofort zu erkennen. Die Häufigkeit der Reluxation aber ist ausschließlich abhängig vom Ausgangsbefund, von der durchgeführten Operation und den Maßnahmen der Retention. Das MRT kann die Reluxation nicht verhindern. Es kann sie allenfalls früh erkennen. Was ist aber früh? Soll nach jeder geschlossenen und offenen Reposition nach 1,3, 7 und 14 Tagen eine Kernspintomographie durchgeführt werden? Wo besteht noch die Indikation zur Sonographie im Gipsverband, die durchaus als erfolgreich beschrieben wurde oder auch zur konventionellen Röntgendiagnostik? Franke et al. beziehen sich darauf, daß konventionelle Röntgenbilder entweder technisch schwierig oder vom Informationsgehalt unbefriedigend seien. Bei ihren Untersuchungen sind die Aussagen der Magnet-Resonanz-Tomographie aber ebenfalls bei 5 von 34 untersuchten Kindern nur eingeschränkt beurteilbar. Dies entspricht einer Rate von $15 \%$, die bei der Beurteilung des Röntgenbildes durch einen erfahrenen Orthopäden etwa in gleicher Höhe sein dürfte.

Sicher ist der Aussage zuzustimmen, daß die computertomographische Untersuchung der reponierten Säuglingshüfte allein wegen der Strahlenbelastung der Vergangenheit angehört. Unterbewertet ist jedoch die Arthrographie. Sie spielt eine nicht zu vernachlässigende Rolle im Behandlungskonzept der kongenitalen Hüftgelenks- 
luxation. Tönnis hat zu Recht unterstrichen, wie wichtig es sei, den Hüftkopf unter Sicht ,in der Hüftpfanne einzuparken, ebenso wie ein Auto mit Licht in die Garage zu fahren". Bei dieser Untersuchung weiß der behandelnde Orthopäde, wo die Instabilitätszonen des Hüftgelenkes liegen, wo sich Grenzen der funktionellen Behandlung aufdrängen und wo die Stabilisierung in bestimmter Position erforderlich ist. Wenn dieser Befund als Engramm gespeichert wird und die Behandlung in einer Hand bleibt, so reicht in der Regel die einmalige klinische und später durchgeführte röntgenologische Untersuchung. um eine Positionskontrolle des Hüftgelenkes vorzunehmen (Niethard 1989)

Es ist erfreulich. wenn die Autoren feststellen. daß für die Routinediagnostik der Hüftdysplasie und Hüftluxation nach wie vor die Sonographie der goldene Standard ist (nach den Leitlinien des sonographischen Screenings immer zusammen mit der klinischen Untersuchung!) Dies muß zunächst auch noch für die röntgenologische Untersuchung der Hüfte gelten. denn sie erlaubt die zusammenfassende Beurteilung des Hüftgelenkes durch den Orthopäden, der sich dieses Gelenkes angenommen hat. Der Einsatz der Magnet-Resonanz-Tomographie kann daher noch nicht als Basisdiagnostik bei der Therapiekontrolle auch nicht nach Reposition der kongenitalen Hüftgelenksluxation angesehen werden.

F. U. Niethard, Aachen

\section{Literatur}

Bos, C. F. A., J. L. Bloem, W. R. Obermann, P. M. Rozing: Magnetic resonance imaging in congenital dislocation of the hip. J. Bone and Joint Surg. 70-B (1988) 174-178

Catterall. A.: The early diagnosis of congenital dislocation of the hip. J. Bone Joint Surg. 76-B (1994) 515-516

Franke, J., D. Lazovic. J. Lotz. O. Rühmann. C. J. Wirth: Die MagnetResonanz-Tomographie als Therapiekontrolle nach Repositionsbehandlung bei kongenitaler Hüftluxation. Z. Orthop. 136 (1998) 205-209

Harcke. H. T.. L. E. Grissom: Performing dynamic sonography of the infant hip. AJR 155 (1990) 837-844

Niethard, F. U.: Röntgenologische Untersuchung der Säuglings- und Kleinkinderhüfte. Z. Orthop. 128 (1990) 357-360

Tönnis, D.: Die angeborene Hüftdysplasie und Hüftluxation. Springer. Berlin-Heidelberg-New York 1984

Wirth. Th. M. Haake, R. Hahn-Rinn, E. Walthers: Die Magnet-Resonanz-Tomographic in der Diagnostik und Therapiekontrolle von $\mathrm{Pa}$ tienten mit kongenitaler Hüftdysplasie- und -luxation. Z. Orthop. 136 (1998) 210-214 